\title{
General Solutions for Axisymmetric Elasticity Problems of Elastic Half Space using Hankel Transform Method
}

\author{
Charles Chinwuba Ike \\ Dept of Civil Engineering \\ Enugu State University of Science \& Technology, Enugu State, Nigeria. \\ ikecc2007@yahoo.com
}

\begin{abstract}
In this work, the Hankel transform method was used to obtain general solutions for stress and displacement fields in semi-infinite linear elastic, isotropic soil under axisymmetric load. Hankel transformation of the governing equations in a stress-based formulation was used to find the Love stress function. Hankel transforms were also applied to the stress and displacement fields to obtain general solutions for the stresses and displacements. The general solutions obtained were used to solve the particular axisymmetric elasticity problem of Boussinesq. The solutions obtained were exactly the same as the solutions obtained by Boussinesq who solved the problem using Boussinesq stress potential functions.
\end{abstract}

Keywords: Hankel transform method, axisymmetric elasticity problem, Love stress function, stress fields, displacement fields, Boussinesq problem

\section{INTRODUCTION / AXISYMMETRIC ELASTICITY PROBLEMS OF SEMI-INFINITE SOIL}

Axisymmetric elasticity problems of semi-infinite soil are problems of finding the stress and displacement fields in soil masses that extend infinitely in the radial coordinate directions, but are bounded from one side with a horizontal boundary surface when the loads are applied on the surface, in such a way as to produce a circular symmetry of the state of stress about a vertical axis which usually corresponds to the vertical axis of symmetry of the applied load. Typical axisymmetrical elasticity problems of geotechnical engineering are the problems of stress and displacement fields in semi-infinite linear elastic, isotropic soil masses due to point loads applied at a point on the soil surface. uniformly distributed load of known intensity applied on a circular area on the soil surface.

Axisymmetric elasticity problems of the half space are commonly encountered in solid and soil mechanics. In geotechnical engineering such problems find applications in the computation of elastic settlements of foundations due to point loads and distributed symmetrical loads applied over loaded areas that ensure symmetry of stress fields [1. 2]. Boussinesq's stress distribution theory, which is based on the mathematical theory of elasticity provides the simplest case of axisymmetrical elasticity problem. In the Boussinesq problem, a single vertical point load is applied at a point on the horizontal boundary surface (ground surface) of a solid assumed to be homogeneous, linear elastic, isotropic and semi-infinite. The vertical axis of load application is the axis of symmetry. The Boussinesq problem is a fundamental problem in soil/solid mechanics and the solutions are also fundamental Green functions that are vital in constructing solutions for distributed loads applied over given areas on the boundary of the semi-infinite soil/solid [3, 4, 5]. Another axisymmetric problem common in soil mechanics and foundation engineering is the problem of finding stress fields and displacements due to uniformly distributed loads applied over circular foundation areas. In this case, the center of the circular foundation is the vertical axis of symmetry. The fundamental assumptions of the Boussinesq's problem are as follows $[5,6]$ : 
the soil is linear elastic, homogeneous, isotropic, semi-infinite; which means it extends infinitely in all directions from the horizontal boundary surface.

the soil material obeys the generalized three dimensional Hooke's laws of linear elasticity.

(iii) originally, prior to the application of the single concentrated load, the soil is not subject to any other stress

(iv) there is continuity of stress

(v) the stress distribution from the externally applied point load is independent of the type of material of which the soil is constituted. The change in volume of the soil due to the application of the stress is disregarded.

the stresses are distributed symmetrically with respect to the axis of symmetry of the problem.

Generally, axisymmetric problems of elasticity belong to the mathematical theory of elasticity, and are solved by simultaneous consideration of the differential equations of equilibrium, the geometric (kinematic relations) and the material constitutive laws subject to the boundary conditions of the problem. The number and complexity of equations involved in a rigorous solution are quite unwieldly and pose mathematical rigors in the analysis [7]. Two basic methods are usually used in the formulation of elasticity problems, namely, displacement-based methods and stress-based methods. In displacement based formulation, the three requirements of kinematics, material constitutive laws and equilibrium are solved such that displacements become the primary unknowns. This has the benefit of reducing the number of equations. The displacement formulation was presented by Navier and Lamé. Stress-based formulations express the three requirements simultaneously by expressing them such that stresses become the primary unknown variables [8, 9, 10]. Stressbased formulations were presented by Michell and Beltrami. The advantage is the reduction in the number of governing equations to be solved. The paper uses the stress formulation method. Stress functions have been developed by many researchers to solve the elasticity problems formulated in terms of stress by Airy, Maxwell, Morera, and Love [4]. Ike [11] derived from first principles, a stress function for three dimensional axially symmetric elasticity problems involving linear elastic, isotropic, homogeneous materials. In the process the fifteen governing partial differential equations of isotropic linear elasticity were reduced to the solution of the biharmonic problem in terms of the stress function, thus simplifying the elasticity problem; and the solution process. The stress function derived by Ike [11] was found to be identical with the Love stress function. Ike successfully applied his derived stress function to obtain solutions for displacement fields and stress fields for the Boussinesq problem; and obtained results that were exactly identical with the results by Boussinesq who used Boussinesq potential functions.

Love stress function has been adopted in this work because it is ideally suited for axisymmetric elasticity problems of the half space. Love stress functions are constructed on the condition that such stress function should be biharmonic functions. In this paper, the Hankel transform method which is an integral transformation technique is applied to obtain general solutions to the general axisymmetric elasticity problem of soil idealized as an elastic half space.

Onah et al [12] applied the Fourier transform method to the determination of stresses induced by infinitely long line loads on semi-infinite, homogeneous elastic soil. Airy's stress potential functions of the Cartesian coordinates system were used to express the governing equations of plane strain elasticity for a semiinfinite homogeneous soil as a biharmonic problem; which was solved using exponential Fourier transform technique. Their solutions were identical with Flammant's solutions and solutions found in the technical literature. Nwoji et al [13] used the Green and Zerna displacement potential function method to solve the three dimensional small deformation axially symmetric problem of the theory of elasticity involving a point load $P$ acting at a point, designated the origin, on the boundary of a linear elastic, homogeneous soil mass of semiinfinite extent. The displacement functions were used to simplify the problem to one of finding the potential function that satisfied the loading boundary conditions. Ike et al [14] used the Trefftz potential function method to solve the three dimensional axisymmetric problem of elasticity for a point load $P$ acting at the origin on the boundary of a linear elastic homogenous soil mass of semi-infinite extent. The Trefftz potential function method simplified the elasticity problem to finding a harmonic function of the space coordinates that satisfies the boundary conditions.

\section{RESEARCH AIM AND OBJECTIVES}

The aim of this research work is to apply the Hankel transform method to obtain the general solutions for the axisymmetric elasticity problems of elastic half-space. The objectives include: 
(i) to apply the Hankel transform method to the governing partial differential equation of the stress formulation of axisymmetric elasticity problems and obtain the solutions for stress potential functions that satisfy bounded conditions.

(ii) to apply the Hankel transform method to the stress and displacement fields, and hence obtain general expressions for the stress and displacement fields in Hankel transform space, and in the physical domain space.

(iii) to apply the general solutions obtained to solve the Boussinesq problem.

\section{THEORETICAL FRAMEWORK}

Axisymmetric elasticity problems of the elastic half space satisfy simultaneously the fundamental equations of elasticity, namely the differential equations of equilibrium, the generalised Hooke's stress-strain laws and the Cauchy strain-displacement relations. The differential equations of elasticity for axisymmetric problems are:

$\frac{1}{r} \frac{\partial}{\partial r} r \sigma_{r r}+\frac{\partial}{\partial z} \tau_{r z}-\frac{\sigma_{\theta \theta}}{r}+F_{r}=0$

$\frac{1}{r} \frac{\partial}{\partial r} r \tau_{z r}+\frac{\partial}{\partial z} \sigma_{z z}+F_{z}=0$

where $\sigma_{r r}$ is the radial stress, $\sigma_{z z}$ is the stress in the $z$ direction, $\sigma_{\theta \theta}$ is the circumferential stress, $\tau_{r z}=\tau_{z r}$ is the shear stress, $F_{r}$ is the body force in the radial direction, $F_{z}$ is the body force in the $z$ direction. In the absence of body forces, the differential equations of static equilibrium become:

$$
\begin{aligned}
& \frac{\partial \sigma_{r r}}{\partial r}+\frac{\partial \tau_{z r}}{\partial z}+\frac{\sigma_{r r}-\sigma_{\theta \theta}}{r}=0 \\
& \frac{\partial \sigma_{z z}}{\partial z}+\frac{\partial \tau_{z r}}{\partial z}+\frac{\tau_{z r}}{r}=0
\end{aligned}
$$

The stress-strain relations are given by:

$$
\begin{aligned}
& \sigma_{r r}=2 G \varepsilon_{r r}+\lambda \varepsilon_{v} \\
& \sigma_{z z}=2 G \varepsilon_{z z}+\lambda \varepsilon_{v} \\
& \sigma_{\theta \theta}=2 G \varepsilon_{\theta \theta}+\lambda \varepsilon_{v} \\
& \tau_{r z}=G \gamma_{r z} \\
& \tau_{\theta z}=0 \\
& \tau_{\theta r}=0
\end{aligned}
$$

or by

$$
\begin{aligned}
& \varepsilon_{r r}=\frac{1}{E}\left(\sigma_{r r}-\mu\left(\sigma_{\theta \theta}+\sigma_{z z}\right)\right) \\
& \varepsilon_{\theta \theta}=\frac{1}{E}\left(\sigma_{\theta \theta}-\mu\left(\sigma_{r r}+\sigma_{z z}\right)\right) \\
& \varepsilon_{z z}=\frac{1}{E}\left(\sigma_{z z}-\mu\left(\sigma_{r r}+\sigma_{\theta \theta}\right)\right) \\
& \gamma_{z r}=\gamma_{r z}=\frac{\tau_{r z}}{G} \\
& \gamma_{\theta z}=0 \\
& \gamma_{\theta r}=0
\end{aligned}
$$


where $\varepsilon_{v}$ is the volumetric strain, $G$ is the shear modulus, $\lambda$ is the Lamé's constant, $\varepsilon_{r r}$ is the radial strain, $\varepsilon_{z z}$ is the vertical strain, $\varepsilon_{\theta \theta}$ is the circumferential strain, $\gamma_{r z}$ is the shear strain in the $r z$ direction, $\gamma_{\theta z}$ is the shear strain in the $\theta z$ direction and $\gamma_{\theta r}$ is the shear strain in the $r \theta$ direction.

The geometric or kinematic (strain-displacement) relations for small deformation assumptions are:

$$
\begin{aligned}
& \varepsilon_{r r}=\frac{\partial u_{r}}{\partial r} \\
& \varepsilon_{r r}=\frac{\partial w}{\partial z}=\frac{\partial u_{z}}{\partial z} \\
& \varepsilon_{\theta \theta}=\frac{u_{r}}{r} \\
& \gamma_{r z}=\frac{\partial u_{r}}{\partial z}+\frac{\partial u_{z}}{\partial r}=\frac{\partial u_{r}}{\partial z}+\frac{\partial w}{\partial r}
\end{aligned}
$$

where $u_{r}$ is the radial displacement, $u_{z}$ or $w$ is the vertical displacement.

The compatibility equations in terms of stress are given by:

$$
\begin{aligned}
& \nabla^{2} \sigma_{r r}-\frac{2}{r^{2}}\left(\sigma_{r r}-\sigma_{\theta \theta}\right)+\frac{1}{1+\mu} \frac{\partial^{2} \theta}{\partial r^{2}}=0 \\
& \nabla^{2} \sigma_{\theta \theta}-\frac{2}{r^{2}}\left(\sigma_{r r}-\sigma_{\theta \theta}\right)+\frac{1}{1+\mu} \frac{1}{r} \frac{\partial \theta}{\partial r}=0 \\
& \nabla^{2} \sigma_{z z}+\frac{1}{1+\mu} \frac{\partial^{2} \theta}{\partial r^{2}}=0 \\
& \nabla^{2} \tau_{z r}-\frac{\tau_{z r}}{r}+\frac{1}{1+\mu} \frac{\partial^{2} \theta}{\partial r \partial z}=0 \\
& \text { where } \quad \theta=\sigma_{r r}+\sigma_{\theta \theta}+\sigma_{z z} \\
& \text { and } \quad \nabla^{2}=\frac{\partial^{2}}{\partial r^{2}}+\frac{1}{r} \frac{\partial}{\partial r}+\frac{\partial^{2}}{\partial z^{2}}
\end{aligned}
$$

Stress functions have been developed to solve the stress compatibility operations. Stress functions are scalar fields developed to mathematically satisfy the stress compatibility equations and the differential equations of equilibrium.

\section{GOVERNING EQUATIONS}

Axisymmetric elasticity problems of the semi-infinite linearly elastic soil in the region $-\infty \leq r \leq \infty$, $0 \leq z \leq \infty$ are governed by the biharmonic equation in terms of the stress function $\varphi(r, z)$ given by [3]:

$$
\begin{aligned}
& \nabla^{4} \varphi(r, z)=\nabla^{2} \nabla^{2} \varphi(r, z)=0 \\
& -\infty \leq r \leq \infty, 0 \leq z \leq \infty
\end{aligned}
$$

where $r$ is the radical coordinate variable, $z$ is the depth coordinate variable, and $\nabla^{2}$ is the Laplacian in terms of the cylindrical coordinates, $\nabla^{4}$ is the biharmonic differential operator in terms of the cylindrical coordinates system.

Thus, the governing equation is 


$$
\nabla^{4} \varphi(r, z)=\left(\frac{\partial^{2}}{\partial r^{2}}+\frac{1}{r} \frac{\partial}{\partial r}+\frac{\partial^{2}}{\partial z^{2}}\right)\left(\frac{\partial^{2}}{\partial r^{2}}+\frac{1}{r} \frac{\partial}{\partial r}+\frac{\partial^{2}}{\partial z^{2}}\right) \varphi(r, z)=0
$$

The stress potential function $\varphi(r, z)$ for axisymmeric elasticity problems of the elastic half space was defined by Love in terms of the stress field and displacement fields as:

$$
\begin{aligned}
& \sigma_{z z}=\frac{\partial}{\partial z}\left((3 \lambda+4 G) \nabla^{2} \varphi-2(\lambda+G) \frac{\partial^{2} \varphi}{\partial z^{2}}\right) \\
& \tau_{r z}=\frac{\partial}{\partial r}\left((\lambda+2 G) \nabla^{2} \varphi-2(\lambda+G) \frac{\partial^{2} \varphi}{\partial z^{2}}\right) \\
& w=\frac{\lambda+2 G}{G} \nabla^{2} \varphi-\frac{\lambda+G}{G} \frac{\partial^{2} \varphi}{\partial z^{2}} \\
& u=-\frac{\lambda+G}{G} \frac{\partial^{2} \varphi}{\partial r \partial z}
\end{aligned}
$$

where $\lambda$ is the elastic property called the Lamé constant, $G$ is the shear modulus of elasticity, $\sigma_{z z}$ is the vertical stress field, $\tau_{r z}$ is the shear stress field in the $r z$ direction, $u$ is the displacement field component in the radial direction and $w$ is the displacement field component in the $z$ direction. $G$ and $\lambda$ are defined as:

$$
\begin{aligned}
& G=\frac{E}{2(1+\mu)} \\
& \lambda=\frac{\mu E}{(1+\mu)(1-2 \mu)}=\frac{2 \mu G}{1-2 \mu}
\end{aligned}
$$

where $\mu$ is the Poisson's ratio and $E$ is the Young's modulus of elasticity.

\section{METHODOLOGY / APPLICATION OF THE HANKEL TRANSFORM METHOD}

Applying the Hankel transformation to both sides of the governing partial differential equation, we obtain,

$$
\int_{0}^{\infty} \nabla^{4} \varphi(r, z) r J_{0}(k r) d r=0
$$

where $J_{0}(k r)$ is the Bessel function and $k$ is the parameter of the Hankel transform.

Expanding the biharmonic operator,

$$
\begin{aligned}
& \int_{0}^{\infty}\left(\frac{\partial^{2}}{\partial r^{2}}+\frac{1}{r} \frac{\partial}{\partial r}+\frac{\partial^{2}}{\partial z^{2}}\right)\left(\frac{\partial^{2} \varphi}{\partial r^{2}}+\frac{1}{r} \frac{\partial \varphi}{\partial r}+\frac{\partial^{2} \varphi}{\partial z^{2}}\right) r J_{0}(k r) d r=0 \\
& \text { Let } \beta=\frac{\partial^{2} \varphi}{\partial r^{2}}+\frac{1}{r} \frac{\partial \varphi}{\partial r}+\frac{\partial^{2} \varphi}{\partial z^{2}}=\nabla^{2} \varphi(r, z)
\end{aligned}
$$

Then,

$$
\begin{aligned}
& \int_{0}^{\infty}\left(\frac{\partial^{2}}{\partial r^{2}}+\frac{1}{r} \frac{\partial}{\partial r}+\frac{\partial^{2}}{\partial z^{2}}\right) \beta r J_{0}(k r) d r=0 \\
& \int_{0}^{\infty}\left(\frac{\partial^{2} \beta}{\partial r^{2}}+\frac{1}{r} \frac{\partial \beta}{\partial r}+\frac{\partial^{2} \beta}{\partial z^{2}}\right) r J_{0}(k r) d r=0
\end{aligned}
$$


$\int_{0}^{\infty}\left(\frac{\partial^{2} \beta}{\partial r^{2}}+\frac{1}{r} \frac{\partial \beta}{\partial r}\right) r J_{0}(k r) d r+\int_{0}^{\infty} \frac{\partial^{2} \beta}{\partial z^{2}} r J_{0}(k r) d r=0$

Simplifying,

$\int_{0}^{\infty}\left(\frac{\partial^{2} \beta}{\partial r^{2}}+\frac{1}{r} \frac{\partial \beta}{\partial r}\right) r J_{0}(k r) d r+\frac{\partial^{2}}{\partial z^{2}} \int_{0}^{\infty} \beta r J_{0}(k r) d r=0$

Noting that

$\left(\frac{\partial^{2} \beta}{\partial r^{2}}+\frac{1}{r} \frac{\partial \beta}{\partial r}\right) r=r \frac{\partial^{2} \beta}{\partial r^{2}}+\frac{\partial \beta}{\partial r}$

$\left(\frac{\partial^{2} \beta}{\partial r^{2}}+\frac{1}{r} \frac{\partial \beta}{\partial r}\right) r=r \frac{\partial}{\partial r} \frac{\partial \beta}{\partial r}+\frac{\partial}{\partial r} \beta$

$\left(\frac{\partial^{2} \beta}{\partial r^{2}}+\frac{1}{r} \frac{\partial \beta}{\partial r}\right) r=\frac{\partial}{\partial r}\left(r \frac{\partial \beta}{\partial r}\right)$

We thus have,

$\int_{0}^{\infty} \frac{\partial}{\partial r}\left(r \frac{\partial \beta}{\partial r}\right) J_{0}(k r) d r+\frac{\partial^{2}}{\partial z^{2}} \int_{0}^{\infty} \beta r J_{0}(k r) d r=0$

Let $\quad \bar{\beta}=\int_{0}^{\infty} \beta r J_{0}(k r) d r$

where $\bar{\beta}$ is the Hankel transform of $\beta$.

Then

$\frac{\partial^{2}}{\partial z^{2}} \bar{\beta}+\int_{0}^{\infty} \frac{\partial}{\partial r}\left(r \frac{\partial \beta}{\partial r}\right) J_{0}(k r) d r=0$

We evaluate the integral in the Equation (47) by using integration by parts

$\int_{0}^{\infty} \frac{\partial}{\partial r}\left(r \frac{\partial \beta}{\partial r}\right) J_{0}(k r) d r=\left[r \frac{\partial \beta}{\partial r} J_{0}(k r)\right]_{0}^{\infty}-\int_{0}^{\infty} r \frac{\partial \beta}{\partial r} \frac{\partial}{\partial r}\left(J_{0} k r\right) d r$

The, Equation (48) becomes:

$\frac{\partial^{2} \beta}{\partial z^{2}}+\left[r \frac{\partial \beta}{\partial r} J_{0}(k r)\right]_{0}^{\infty}-\int_{0}^{\infty} r \frac{\partial \beta}{\partial r}\left(\frac{\partial}{\partial r} J_{0}(k r)\right) d r=0$

$\frac{\partial \beta}{\partial r}$ is assumed to be finite for all values of $r$, thus

$\left[r \frac{\partial \beta}{\partial r} J_{0}(k r)\right]_{0}^{\infty}=0$

We then obtain,

$\frac{\partial^{2} \bar{\beta}}{\partial z^{2}}-\int_{0}^{\infty} r \frac{\partial \beta}{\partial r}\left(-k J_{1}(k r)\right) d r=0$ 
$\frac{\partial^{2} \bar{\beta}}{\partial z^{2}}+k \int_{0}^{\infty} r \frac{\partial \beta}{\partial r} J_{1}(k r) d r=0$

The integral is evaluated by integration by parts; and we have:

$$
\begin{aligned}
& \frac{\partial^{2} \bar{\beta}}{\partial z^{2}}+k\left\{\left[\beta J_{1}(k r)\right]_{0}^{\infty}-\int_{0}^{\infty} \beta \frac{\partial}{\partial r} r J_{1}(k r) d r\right\}=0 \\
& \frac{\partial^{2} \bar{\beta}}{\partial z^{2}}+\left[k \beta J_{1}(k r)\right]_{0}^{\infty}-k \int_{0}^{\infty} \beta \frac{\partial}{\partial r} r J_{1}(k r) d r=0
\end{aligned}
$$

Similarly, for finite values of $\beta$ for all $r$,

$$
\left[k \beta J_{1}(k r)\right]_{0}^{\infty}=0
$$

We thus obtain:

$$
\begin{aligned}
& \frac{\partial^{2} \bar{\beta}}{\partial z^{2}}-k \int_{0}^{\infty} \beta k r J_{0}(k r) d r=0 \\
& \frac{\partial^{2} \bar{\beta}}{\partial z^{2}}-k^{2} \int_{0}^{\infty} \beta J_{0}(k r) r d r=0 \\
& \frac{\partial^{2} \bar{\beta}}{\partial z^{2}}-k^{2} \bar{\beta}=0=\left(\frac{\partial^{2}}{\partial z^{2}}-k^{2}\right) \bar{\beta}
\end{aligned}
$$

Using Equation (37), we have

$$
\left(\frac{\partial^{2}}{\partial z^{2}}-k^{2}\right) \int_{0}^{\infty}\left(\frac{\partial^{2} \varphi}{\partial r^{2}}+\frac{1}{r} \frac{\partial \varphi}{\partial r}+\frac{\partial^{2} \varphi}{\partial z^{2}}\right) r J_{0}(k r) d r=0
$$

By a similar procedure, we obtain,

$$
\begin{aligned}
& \int_{0}^{\infty}\left(\frac{\partial^{2} \varphi}{\partial r^{2}}+\frac{1}{r} \frac{\partial \varphi}{\partial r}+\frac{\partial^{2} \varphi}{\partial z^{2}}\right) r J_{0}(k r) d r=\int_{0}^{\infty}\left(\frac{\partial^{2} \varphi}{\partial r^{2}}+\frac{1}{r} \frac{\partial \varphi}{\partial r}\right) r J_{0}(k r) d r+\int_{0}^{\infty} \frac{\partial^{2} \varphi}{\partial z^{2}} r J_{0}(k r) d r \\
& \int_{0}^{\infty}\left(\frac{\partial^{2} \varphi}{\partial r^{2}}+\frac{1}{r} \frac{\partial \varphi}{\partial r}+\frac{\partial^{2} \varphi}{\partial z^{2}}\right) r J_{0}(k r) d r=\int_{0}^{\infty}\left(\frac{\partial^{2} \varphi}{\partial r^{2}}+\frac{1}{r} \frac{\partial \varphi}{\partial r}\right) r J_{0}(k r) d r+\frac{\partial^{2}}{\partial z^{2}} \int_{0}^{\infty} \varphi J_{0}(k r) r d r \\
& \int_{0}^{\infty}\left(\frac{\partial^{2} \varphi}{\partial r^{2}}+\frac{1}{r} \frac{\partial \varphi}{\partial r}+\frac{\partial^{2} \varphi}{\partial z^{2}}\right) r J_{0}(k r) d r=\left(\frac{\partial^{2}}{\partial z^{2}}-k^{2}\right) \int_{0}^{\infty} \varphi(r, z) r J_{0}(k r) d r \\
& \int_{0}^{\infty}\left(\frac{\partial^{2} \varphi}{\partial r^{2}}+\frac{1}{r} \frac{\partial \varphi}{\partial r}+\frac{\partial^{2} \varphi}{\partial z^{2}}\right) r J_{0}(k r) d r=\left(\frac{\partial^{2}}{\partial z^{2}}-k^{2}\right) \Phi(k, z)
\end{aligned}
$$

where $\Phi(k, z)=\int_{0}^{\infty} \varphi(r, z) r J_{0}(k r) d r$

and $\Phi(k, z)$ is the Hankel transform of the stress function $\varphi(r, z)$ for the axisymmetric elasticity problem of the elastic half space.

Hence, 


$$
\begin{aligned}
& \int_{0}^{\infty} \nabla^{4} \varphi(r, z) r J_{0}(k r) d r=\left(\frac{\partial^{2}}{\partial z^{2}}-k^{2}\right)\left(\frac{\partial^{2}}{\partial z^{2}}-k^{2}\right) \int_{0}^{\infty} \varphi(r, z) r J_{0}(k r) d r \\
& \int_{0}^{\infty} \nabla^{4} \varphi(r, z) r J_{0}(k r) d r=\left(\frac{\partial^{2}}{\partial z^{2}}-k^{2}\right)^{2} \Phi(k, z)=0
\end{aligned}
$$

We solve the fourth order differential equation in $\Phi(k, z)$ using the method of undetermined parameters by assuming the solution for $\Phi(k, z)$ in the exponential form:

Let $\Phi(k, z)=\exp m z$

where $m$ is an unknown parameter we seek to find.

This yields the characteristic equation:

$\left(m^{2}-k^{2}\right)^{2}=0$

Solving $m=-k$, (twice)

$m=k$ (twice)

The general solution is

$\Phi(k, z)=\left(c_{1}+c_{2} z\right) e^{-k z}+\left(c_{3}+c_{4} z\right) e^{k z}$

where $c_{1}, c_{2}, c_{3}$ and $c_{4}$ are the four constants of integration.

For bounded solutions, $\Phi(k, z)$ should be finite as $z \rightarrow \infty$. Hence

$c_{3}=c_{4}=0$

Thus, for bounded solutions,

$\Phi(k, z)=\left(c_{1}+c_{2} z\right) e^{-k z}$

\section{RESULtS}

\section{Stress fields}

The Hankel transform is applied to both sides of the Love stress function expression for $\sigma_{z z}$ to obtain

$$
\begin{aligned}
\int_{0}^{\infty} \sigma_{z z}(r, z) & J_{0}(k r) r d r=\bar{\sigma}_{z z}(k, z) \\
= & \int_{0}^{\infty} \frac{\partial}{\partial z}\left((3 \lambda+4 G) \nabla^{2} \varphi-2(\lambda+G) \frac{\partial^{2} \varphi}{\partial z^{2}}\right) r J_{0}(k r) d r
\end{aligned}
$$

where $\bar{\sigma}_{z z}(k, z)$ is the Hankel transform of $\sigma_{z z}(r, z)$

Simplification yields,

$$
\begin{aligned}
& \bar{\sigma}_{z z}(k, z)=(3 \lambda+4 G) \int_{0}^{\infty} \frac{\partial}{\partial z} \nabla^{2} \varphi J_{0}(k r) r d r-2(\lambda+G) \int_{0}^{\infty} \frac{\partial}{\partial z} \frac{\partial^{2} \varphi}{\partial z^{2}} r J_{0}(k r) d r \\
& \bar{\sigma}_{z z}(k, z)=(3 \lambda+4 G) \frac{\partial}{\partial z} \int_{0}^{\infty} \nabla^{2} \varphi J_{0}(k r) r d r-2(\lambda+G) \frac{\partial}{\partial z} \int_{0}^{\infty} r \frac{\partial^{2} \varphi}{\partial z^{2}} J_{0}(k r) d r \\
& \bar{\sigma}_{z z}(k, z)=(3 \lambda+4 G) \frac{\partial}{\partial z}\left(\frac{\partial^{2}}{\partial z^{2}}-k^{2}\right) \Phi(k, z)-2(\lambda+G) \frac{\partial^{3}}{\partial z^{3}} \Phi(k, z)
\end{aligned}
$$




$$
\begin{aligned}
& \bar{\sigma}_{z z}(k, z)=(3 \lambda+4 G) \frac{\partial^{3} \Phi}{\partial z^{3}}-(3 \lambda+4 G) k^{2} \frac{\partial \Phi}{\partial z}-2(\lambda+G) \frac{\partial^{3} \Phi}{\partial z^{3}} \\
& \bar{\sigma}_{z z}(k, z)=(\lambda+2 G) \frac{\partial^{3} \Phi}{\partial z^{3}}-(3 \lambda+4 G) k^{2} \frac{\partial \Phi}{\partial z}
\end{aligned}
$$

The vertical stress field in the physical space domain is obtained by inversion of $\bar{\sigma}_{z z}(k, z)$ to obtain:

$$
\begin{aligned}
& \sigma_{z z}(r, z)=\int_{0}^{\infty}\left((\lambda+2 G) \frac{\partial^{3} \Phi}{\partial z^{3}}-(3 \lambda+4 G) k^{2} \frac{\partial \Phi}{\partial z}\right) k J_{0}(k r) d k \\
& \sigma_{z z}(r, z)=\int_{0}^{\infty} \frac{2 G(1-\mu)}{1-2 \mu} k \frac{\partial^{3} \Phi}{\partial z^{3}} J_{0}(k r) d k-\int_{0}^{\infty} \frac{2 G(2-\mu)}{1-2 \mu} k^{3} \frac{\partial \Phi}{\partial z} J_{0}(k r) d k \\
& \sigma_{z z}(r, z)=\frac{2 G}{1-2 \mu}\left\{\int_{0}^{\infty}(1-\mu) k \frac{\partial^{3} \Phi}{\partial z^{3}} J_{0}(k r) d k-\int_{0}^{\infty}(2-\mu) k^{3} \frac{\partial \Phi}{\partial z} J_{0}(k r) d k\right\}
\end{aligned}
$$

\section{Shear stress fields}

Application of the Hankel transformation to the Love stress function expression for the shear stress field $\tau_{r z}(r, z)$ yields

$$
\begin{aligned}
& \int_{0}^{\infty} \tau_{r z} J_{1}(k r) r d r=\bar{\tau}_{r z}(k, z)=\int_{0}^{\infty} \frac{\partial}{\partial r}\left((\lambda+2 G) \nabla^{2} \varphi-2(\lambda+G) \frac{\partial^{2} \varphi}{\partial z^{2}}\right) J_{1}(k r) r d r \\
& \bar{\tau}_{r z}(k, z)=(\lambda+2 G) \int_{0}^{\infty} r \frac{\partial}{\partial r} \nabla^{2} \varphi J_{1}(k r) d r-2(\lambda+G) \int_{0}^{\infty} r \frac{\partial}{\partial r} \frac{\partial^{2}}{\partial z^{2}} \varphi J_{1}(k r) d r \\
& \bar{\tau}_{r z}(k, z)=(\lambda+2 G) \int_{0}^{\infty} r \frac{\partial}{\partial r} \nabla^{2} \varphi J_{1}(k r) d r-2(\lambda+G) \int_{0}^{\infty} r \frac{\partial^{3} \varphi}{\partial z^{2} \partial r} J_{1}(k r) d r
\end{aligned}
$$

Using integration by parts,

$$
\begin{aligned}
& \bar{\tau}_{r z}(k, z)=(\lambda+2 G)\left\{\left[r J_{1}(k r) \nabla^{2} \varphi\right]_{0}^{\infty}-\int_{0}^{\infty} \nabla^{2} \varphi \frac{\partial}{\partial r}\left(r J_{1}(k r)\right) d r\right\} \\
& -2(\lambda+G) \frac{\partial^{2}}{\partial z^{2}} \int_{0}^{\infty} r \frac{\partial \varphi}{\partial r} J_{1}(k r) d r \\
& \bar{\tau}_{r z}(k, z)=-(\lambda+2 G) k \int_{0}^{\infty} \nabla^{2} \varphi r J_{0}(k r) d r \\
& +2(\lambda+G) \frac{\partial^{2}}{\partial z^{2}}\left\{k \int_{0}^{\infty} r \varphi J_{0}(k r) d r-\left[\varphi r J_{1}(k r)\right]_{0}^{\infty}\right\} \\
& \bar{\tau}_{r z}(k, z)=-(\lambda+2 G) k\left(\frac{\partial^{2}}{\partial z^{2}}-k^{2}\right) \Phi+2(\lambda+G) k \frac{\partial^{2} \Phi}{\partial z^{2}} \\
& \bar{\tau}_{r z}(k, z)=\lambda k \frac{\partial^{2} \Phi}{\partial z^{2}}+(\lambda+2 G) k^{3} \Phi
\end{aligned}
$$


This Equation (89) is the general solution for $\tau_{r z}$ in the Hankel transform space.

$$
\begin{aligned}
& \bar{\tau}_{r z}(k, z)=\frac{2 \mu G}{1-2 \mu} k \frac{\partial^{2} \Phi}{\partial z^{2}}+\frac{2 G(1-\mu)}{1-2 \mu} k^{3} \Phi \\
& \bar{\tau}_{r z}(k, z)=\frac{2 G}{1-2 \mu}\left\{\mu k \frac{\partial^{2} \Phi}{\partial z^{2}}+(1-\mu) k^{3} \Phi\right\}
\end{aligned}
$$

The shear stress field in the physical space domain of the soil is obtained by inversion as

$$
\begin{aligned}
& \tau_{r z}(r, z)=\int_{0}^{\infty} k\left(\lambda \frac{\partial^{2} \Phi}{\partial z^{2}}+(\lambda+2 G) k^{3} \Phi\right) J_{1}(k r) d k \\
& \tau_{r z}(r, z)=\frac{2 G}{1-2 \mu} \int_{0}^{\infty}\left(\mu k^{2} \frac{\partial^{2} \Phi}{\partial z^{2}}+(1-\mu) k^{4} \Phi\right) J_{1}(k r) d k
\end{aligned}
$$

\section{Displacement field}

Application of the Hankel transformation to the Love stress function expression for the vertical displacement field, we have

$$
\begin{aligned}
& \int_{0}^{\infty} w J_{0}(k r) r d r=\bar{w}(k, z)=\int_{0}^{\infty}\left(\frac{\lambda+2 G}{G} \nabla^{2} \varphi-\frac{\lambda+G}{G} \frac{\partial^{2} \varphi}{\partial z^{2}}\right) J_{0}(k r) r d r \\
& \bar{w}(k, z)=\frac{\lambda+2 G}{G} \int_{0}^{\infty} \nabla^{2} \varphi J_{0}(k r) r d r-\frac{\lambda+G}{G} \int_{0}^{\infty} \frac{\partial^{2} \varphi}{\partial z^{2}} J_{0}(k r) r d r \\
& \bar{w}(k, z)=\left(\frac{\lambda+2 G}{G}\right)\left(\frac{\partial^{2}}{\partial z^{2}}-k^{2}\right) \int_{0}^{\infty} r \varphi(r, z) J_{0}(k r) d r \\
& \overline{\bar{w}}(k, z)=\left(\frac{\lambda+2 G}{G}\right)\left(\frac{\partial^{2}}{\partial z^{2}}-k^{2}\right) \Phi-\frac{\lambda+G}{\partial z^{2}} \int_{0}^{\infty} r \varphi(r, z) J_{0}(k r) d r \\
& \bar{\partial} z^{2} \\
& \bar{w}(k, z)=\frac{\partial^{2} \Phi}{\partial z^{2}}-\frac{\lambda+2 G}{G} k^{2} \Phi \\
& \bar{w}(k, z)=\frac{\partial^{2} \Phi}{\partial z^{2}}-\frac{2(1-\mu)}{1-2 \mu} k^{2} \Phi
\end{aligned}
$$

The general solution for the vertical displacement in the physical domain space variables $w(r, z)$ is obtained by inversion of the Hankel transform as follows:

$$
\begin{aligned}
& w(r, z)=\int_{0}^{\infty} k\left(\frac{\partial^{2} \Phi}{\partial z^{2}}-\frac{\lambda+2 G}{G} k^{2} \Phi\right) J_{0}(k r) d k \\
& w(r, z)=\int_{0}^{\infty}\left(k \frac{\partial^{2} \Phi}{\partial z^{2}}-\frac{\lambda+2 G}{G} k^{3} \Phi\right) J_{0}(k r) d k
\end{aligned}
$$


$w(r, z)=\int_{0}^{\infty}\left(k \frac{\partial^{2} \Phi}{\partial z^{2}}-\frac{2(1-\mu)}{1-2 \mu} k^{3} \Phi\right) J_{0}(k r) d k$

\section{Horizontal displacement}

Applying the Hankel transformation to the Love stress function expression for the horizontal displacement, we obtain:

$$
\begin{aligned}
& \int_{0}^{\infty} u J_{1}(k r) r d r=\bar{u}(k, z)=\int_{0}^{\infty}-\left(\frac{\lambda+G}{G}\right) \frac{\partial^{2} \varphi}{\partial r \partial z} J_{1}(k r) r d r \\
& \bar{u}(k, z)=-\frac{\lambda+G}{G} \int_{0}^{\infty} \frac{\partial^{2} \varphi}{\partial r \partial z} J_{1}(k r) r d r \\
& \bar{u}(k, z)=-\frac{\lambda+G}{G} \frac{\partial}{\partial z} \int_{0}^{\infty} \frac{\partial \varphi}{\partial r} r J_{1}(k r) d r \\
& \bar{u}(k, z)=-\frac{\lambda+G}{G} \frac{\partial}{\partial z}\left\{\left[\varphi r J_{1}(k r)\right]_{0}^{\infty}-\frac{\partial}{\partial r} \int_{0}^{\infty} \varphi \frac{\partial}{\partial r} r J_{1}(k r) d r\right\} \\
& \bar{u}(k, z)=\frac{\lambda+G}{G} k \frac{\partial}{\partial z} \int_{0}^{\infty} \varphi r J_{0}(k r) d r \\
& \bar{u}(k, z)=\frac{\lambda+G}{G} k \frac{\partial \Phi}{\partial z}=\frac{1}{1-2 \mu} k \frac{\partial \Phi}{\partial z}
\end{aligned}
$$

The horizontal displacement in the physical domain space of the problem is obtained by inversion as:

$$
\begin{aligned}
& u(r, z)=\int_{0}^{\infty} \frac{\lambda+G}{G} k \frac{\partial \Phi}{\partial z} k J_{1}(k r) d k \\
& u(r, z)=\frac{\lambda+G}{G} \int_{0}^{\infty} k^{2} \frac{\partial \Phi}{\partial z} J_{1}(k r) d k \\
& u(r, z)=\frac{1}{1-2 \mu} \int_{0}^{\infty} k^{2} \frac{\partial \Phi}{\partial z} J_{1}(k r) d k
\end{aligned}
$$

\section{Particular solutions for the Boussinesq problem}

The Boussinesq problem shown in Figure 1 is now considered as a specific illustration of the general solutions obtained. 


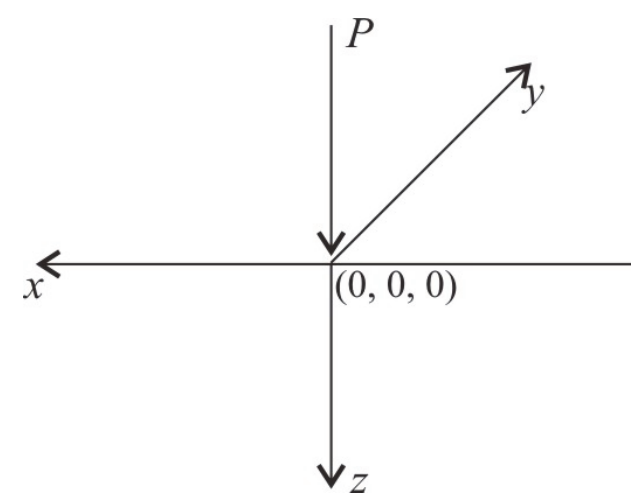

Figure 1: Point load at the origin of a semi-infinite linear elastic soil

$(-\infty \leq x \leq \infty,-\infty \leq y \leq \infty, 0 \leq z \leq \infty$ or $-\infty \leq r \leq \infty, 0 \leq z \leq \infty)$

From Equation (73), by differentiation with respect to $z$,

$$
\begin{aligned}
& \frac{\partial \Phi}{\partial z}=\left(c_{2}(1-k z)-c_{1} k\right) e^{-k z} \\
& \frac{\partial^{2} \Phi}{\partial z^{2}}=\left(c_{2}\left(k^{2} z-2 k\right)+c_{1} k^{2}\right) e^{-k z} \\
& \frac{\partial^{3} \Phi}{\partial z^{3}}=\left(c_{2}\left(3 k^{2}-k^{3} z\right)-c_{1} k^{3}\right) e^{-k z}
\end{aligned}
$$

The boundary conditions are

$$
\tau_{r z}(r, z=0)=0
$$

Therefore

$$
\begin{aligned}
& \int_{0}^{\infty}\left((1-\mu) \nabla^{2}-\frac{\partial^{2}}{\partial z^{2}}\right) \varphi(r, z) r J_{0}(k r) d r=0 \\
& \int_{0}^{\infty}\left(\mu k^{2} \frac{\partial^{2} \Phi}{\partial z^{2}}+(1-\mu) k^{4} \Phi\right) J_{1}(k r) d k=0
\end{aligned}
$$

This is true when

$$
\begin{aligned}
& \mu \frac{d^{2} \Phi}{d z^{2}}+\left.(1-\mu) k^{2} \Phi\right|_{r, z=0}=0 \\
& \mu\left(c_{2}(-2 k)+c_{1} k^{2}\right)+(1-\mu) k^{2} c_{1}=0 \\
& -2 c_{2} \mu k+c_{1} \mu k^{2}+k^{2} c_{1}-\mu k^{2} c_{1}=0 \\
& k^{2} c_{1}-2 c_{2} \mu k=0 \\
& c_{1}=\frac{2 \mu k c_{2}}{k^{2}}=\frac{2 \mu c_{2}}{k}
\end{aligned}
$$

The boundary condition for $\sigma_{z z}$ is

$$
\sigma_{z z}=-\lim _{R \rightarrow 0} \frac{P}{\pi R^{2}}
$$


The Hankel transform of the point load is

$$
\begin{aligned}
& \bar{P}_{0}(k, z)=\lim _{R \rightarrow 0} \int_{0}^{R} \frac{P}{\pi R^{2}} r J_{0}(k r) d r \\
& \bar{P}_{0}(k, z)=\lim _{R \rightarrow 0} \frac{P}{\pi R^{2}} \frac{R}{k} J_{1}(k R)=\frac{-P}{2 \pi}
\end{aligned}
$$

Hence,

$$
\frac{2 G}{1-2 \mu} \int_{0}^{\infty}\left((1-\mu) \frac{\partial^{3} \Phi}{\partial z^{3}}-(2-\mu) k^{2} \frac{\partial \Phi}{\partial z}\right) J_{0}(k r) d r=\int_{0}^{\infty} r P(r) J_{0}(k r) d r=\bar{P}_{0}(k, z)
$$

where $\bar{P}_{0}(k, z)$ is the Hankel transform of the point load

$$
\begin{aligned}
& \left.\frac{2 G}{1-2 \mu}\left((1-\mu) \frac{d^{3} \Phi}{d z^{3}}-(2-\mu) k^{2} \frac{d \Phi}{d z}\right)\right|_{z=0}=\bar{P}_{0}(k, z) \\
& \frac{2 G}{1-2 \mu}\left((1-\mu)\left(3 k^{2} c_{2}-c_{1} k^{3}\right)-(2-\mu) k^{2}\left(c_{2}-c_{1} k\right)\right)=\frac{-P}{2 \pi} \\
& \frac{2 G}{1-2 \mu}\left((3-3 \mu) k^{2} c_{2}-(1-\mu) c_{1} k^{3}-(2-\mu) k^{2} c_{2}+(2-\mu) k^{3} c_{1}\right)=\frac{-P}{2 \pi} \\
& \frac{2 G}{1-2 \mu}\left((1-2 \mu) k^{2} c_{2}+c_{1} k^{3}\right)=\frac{-P}{2 \pi} \\
& \frac{2 G}{1-2 \mu}\left((1-2 \mu) k^{2} c_{2}+\frac{2 \mu}{k} c_{2} k^{3}\right)=\frac{-P}{2 \pi} \\
& \frac{2 G}{1-2 \mu}\left(k^{2} c_{2}\right)=\frac{-P}{2 \pi} \\
& c_{2}=\frac{-P(1-2 \mu)}{2 \pi k^{2} 2 G} \\
& c_{2}=\frac{-P(1-2 \mu)}{4 \pi G k^{2}}=\frac{-P(1-2 \mu)}{2 G 2 \pi k^{2}} \\
& c_{1}=\frac{2 \mu}{k} c_{2}=\frac{2 \mu}{k} \frac{-P(1-2 \mu)}{4 \pi G k^{2}} \\
& c_{1}=\frac{-P \mu(1-2 \mu)}{2 \pi G k^{3}}=\frac{-P \mu(1-2 \mu)}{2 G \cdot \pi k^{3}}
\end{aligned}
$$

\section{Stress fields}

$$
\begin{aligned}
\sigma_{z z}(r, z) & =\frac{2 G}{1-2 \mu}\left\{\int_{0}^{\infty}(1-\mu) k\left(c_{2}\left(3 k^{2}-k^{3} z\right)-c_{1} k^{3}\right) e^{-k z} J_{0}(k r) d k\right. \\
& \left.-\int_{0}^{\infty}(2-\mu) k^{3}\left(c_{2}(1-k z)-c_{1} k\right) e^{-k z} J_{0}(k r) d k\right\}
\end{aligned}
$$




$$
\begin{aligned}
& \sigma_{z z}(r, z)=\frac{2 G}{1-2 \mu}\left\{\int_{0}^{\infty}\left((1-2 \mu) k^{3}+k^{4} z\right) c_{2}+k^{4} c_{1}\right\} e^{-k z} J_{0}(k r) d k \\
& \sigma_{z z}=\frac{2 G}{1-2 \mu}\left\{\int_{0}^{\infty}\left((1-2 \mu) k^{3}+k^{4} z\right) \frac{-P(1-2 \mu)}{4 \pi G k}-\frac{k^{4} P \mu(1-2 \mu)}{2 \pi G k^{3}}\right\} e^{-k z} J_{0}(k r) d k \\
& =\frac{-P}{2 \pi} \int_{0}^{\infty}\left((1-2 \mu) k+k^{2} z+2 k \mu\right) e^{-k z} J_{0}(k r) d k \\
& \sigma_{z z}(r, z)=\frac{-P}{2 \pi} \int_{0}^{\infty}(1+k z) k e^{-k z} J_{0}(k r) d k \\
& \sigma_{z z}(r, z)=\frac{-P}{2 \pi}\left[\frac{\left(2 z^{2}-r^{2}\right) z}{\left(r^{2}+z^{2}\right)^{5 / 2}}+\frac{z}{\left(r^{2}+z^{2}\right)^{3 / 2}}\right] \\
& \sigma_{z z}=\frac{-3 P z^{3}}{2 \pi R^{5}}
\end{aligned}
$$

Similarly,

$$
\begin{aligned}
& \tau_{r z}=-\int_{0}^{\infty} \frac{P k z}{2 \pi} k e^{-k z} J_{1}(k r) d k \\
& \tau_{r z}=\frac{-P}{2 \pi} \int_{0}^{\infty} k^{2} z e^{-k z} J_{1}(k r) d k \\
& \tau_{r z}=\frac{-P z}{2 \pi} \frac{3 z r}{\left(r^{2}+z^{2}\right)^{5 / 2}}=\frac{-3 P z^{2} r}{2 \pi R^{5}} \\
& u=-\int_{0}^{\infty} \frac{P}{4 \pi G}((1-2 \mu)-k z) e^{-k z} J_{1}(k r) d k \\
& u=\frac{-P}{4 \pi G} \int_{0}^{\infty}((1-2 \mu)-k z) e^{-k z} J_{1}(k r) d k \\
& u=\frac{-P}{4 \pi G}\left(\frac{r z}{R^{3}}+\frac{(1-2 \mu) r}{R(R+z)}\right) \\
& u=\frac{-P(1+\mu)}{2 \pi E}\left(\frac{r z}{R^{3}}+\frac{(1-2 \mu) r}{R(R+z)}\right) \\
& w=\frac{P}{4 \pi G}\left(\frac{2(1-\mu)}{R}+\frac{z^{2}}{R^{3}}\right) \\
& u_{z}=w=\int_{0}^{\infty} \frac{P}{4 \pi G}(2(1-\mu)+k z) e^{-k z} J_{0}(k r) d k \\
& w= \\
& 0
\end{aligned}
$$


$w=\frac{P(1+\mu)}{2 \pi E}\left(\frac{2(1-\mu)}{R}+\frac{z^{2}}{R^{3}}\right)$

\section{DISCUSSION}

In this paper, the Hankel transform method has been successfully implemented to obtain general solutions for the axisymmetric elasticity problems of elastic half space. Stress formulation approach was adopted and Love stress functions were used to reduce the mathematically rigorous demands of the simultaneous solution of geometric (kinematic) material constitutive and equilibrium requirements to one of finding a biharmonic stress function $\varphi(r, z)$ in the cylindrical coordinate system that satisfied completely the boundary conditions on stresses as well as the bounded requirements of the solutions. The governing partial differential equation was transformed by the application of Hankel transformation with respect to the radial coordinate variable to a linear ordinary differential equation that is of fourth order. The fourth order equation was solved and the solution for bounded stress fields and deformation fields obtained in the Hankel transform space as Equation (73) for the general case. The Love stress function expressions for the vertical stress fields and the shear stress fields were similarly transformed using the Hankel transform to obtain general solutions in the transformed space variables as Equation (79) for $\bar{\sigma}_{z z}(k, z)$ and Equation (89) for $\bar{\tau}_{r z}(k, z)$. By inversion, general solutions in the physical domain space variables were obtained for $\sigma_{z z}(r, z)$ as Equation (82) and for $\tau_{r z}(r, z)$ as Equation (93). Similarly, the Hankel transform was applied to the Love stress function expression for the horizontal (radial) and vertical displacements, to obtain the general solution for the vertical displacement as Equation (99) in the Hankel transform space and the general solution for the radial (horizontal) displacement as Equation (108) in the Hankel transform space. By inversion, the general solutions in the physical domain space of the problem were obtained as Equation (102) for the vertical displacement and Equation (111) for the radial displacement. The general solutions obtained were now applied to solve the particular axisymmetric elasticity problem originally solved by Boussinesq using Boussinesq stress functions. The boundary conditions of the Boussinesq problem, shown in Equations (115) and (126) were used to find the two integration constants of the Hankel transformd Love stress function as Equation (134) for $c_{2}$ and Equation (136) for $c_{1}$. These constants completely determined the Love stress function for the Boussinesq problem and enabled the determination of the vertical stress field as Equation (143) and the shear stress field as Equation (146). Similarly, the radial displacement was found as Equation (150) and the vertical displacement as Equation (154). The solutions for the stress fields and displacement fields were exactly the same as solutions obtained by Boussinesq who used Boussinesq stress functions. The vertical stress obtained was found to be radially symmetrical. The vertical stress distribution is found to be singular and infinite at the point of application of the point load and decreases with the square of the depth. At any arbitrary depth the distribution of vertical stress on a horizontal plane is bell-shaped.

\section{Conclusions}

The following conclusions can be made:

(i) The Hankel transform method is an effective analytical method for solving axisymmetric elasticity problems of the elastic half space for the general case.

(ii) The Hankel transform method is an effective mathematical technique for solving the axisymmetric elasticity problem of Boussinesq.

(iii) The Hankel transform method simplifies the fourth order biharmonic equation for the Love stress function $\varphi(r, z)$ to a linear ordinary differential equation with constant parameters, which is easier to solve than the original partial differential equation.

(iv) The Love stress function formulation of the general and specific (Boussinesq) axisymmetric elasticity simplified the solution of the problem to finding a scalar field $\varphi(r, z)$ that satisfied the biharmonic equation, the boundary conditions and the bounded requirements of the stress fields and deformation fields. 


\section{REFERENCES}

[1] K.T. Chan "Analytic Methods in Geomechanics," CRC Press Taylor and Francis Group, New York, 2013.

[2] M.H. Sadd "Elasticity Theory, Applications, and Numerics," Third Edition. Elsevier Academic Press, Amsterdam, 2014.

[3] P. Padio-Guidugli and A. Favata "Elasticity for Geotechnicians: A modern exposition of Kelvin, Boussinesq, Flammant, Cerruti, Melan and Mindlin Problems," Solid Mechanics and its applications, Springer, 2014.

[4] A.E. Love "The stress produced in a semi-infinite solid by pressure on part of the boundary," Philosophical transactions of the Royal Society A Vol. 228 No 659-669, pp. 377-420, 1929.

[5] M.J. Boussinesq "Application des potentials a l'etude de l'equilibre et des mouvements des solides elastiques," Paris, GantherVillars, 1885

[6] C.C. Ike "Principles of Soil Mechanics," De-Adroit Innovation, Enugu, 2006.

[7] S.A. Lurie and V.V. Vasilev "The Biharmonic Problem in the Theory of Elasticity," Gorden and Breach Publishers, United States, United Kingdom, 1995.

[8] T.G. Sitharam and R.L. Govinda "Applied Elasticity for Engineers Module: Elastic Solutions and Applications in Geomechanics," 14.139.172.204/nptel/1/CSE/web/ 105108070/module 8/lecture 17.pdf

[9] A. Hazel MATH 350211 Elasticity www.maths.manchester.ac.uk/ahazel/ MATHS. Nov 30, 2015.

[10] D. Palaniappan "A general solution of equations of equilibrium in linear elasticity," Applied Mathematical Modelling, $35 \mathrm{pp}$ 5494-5499, Elsevier, 2011.

[11] C.C. Ike "First Principles Derivation of a Stress Function for Axially Symmetric Elasticity Problems, and Application to Boussinesq Problem," Nigerian Journal of Technology (NIJOTECH), Vol. 36 No. 3, pp.767-772, 2017.

[12] H.N. Onah, N.N. Osadebe, C.C. Ike and C.U. Nwoji "Determination of stresses caused by infinitely long line loads on semiinfinite elastic soils using Fourier Transform Method," Nigerian Journal of Technology (NIJOTECH), Vol. 35, No. 4, pp. 726$731,2016$.

[13] C.U. Nwoji, H.N. Onah, B.O. Mama and C.C. Ike "Solution of the Boussinesq Problems of Half Space using Green and Zerma Displacement Potential Function Method," Electronic Journal of Geotechnical Engineering, (22.10). pp. 4305-4314, 2017.

[14] C.C. Ike, B.O. Mama, H.N. Onah and C.U. Nwoji "Trefftz Harmonic Function Method for Solving Boussinesq Problem," Electronic Journal of Geotechnical Engineering, (22.12). pp. 4589-4601, 2017.

\section{Author Profile}

Engr Dr. Charles C. Ike is a lecturer at the Enugu State University of Science and Technology, Enugu, Nigeria. He holds a PhD in structural engineering, and is a member of the Nigerian Society of Engineers. He is registered with the Council for the Regulation of Engineering in Nigeria (COREN). He has numerous publications in reputable international journals. 